\section{Malignant histiocytosis of lungs and chest wall: A case report of diagnostic conundrum and treatment challenges in a resource-poor setting}

\author{
Jameel Ismail Ahmad, ${ }^{1}$ \\ Ismail Mohammed Inuwa, ${ }^{1}$ \\ Dalha Gwarzo Haliru, ${ }^{2}$ \\ Imam Mohammed Ibrahim ${ }^{3}$ \\ ${ }^{1}$ Division of Cardiothoracic Surgery, \\ Surgery Department, ${ }^{2}$ Department of \\ Hematology, ${ }^{3}$ Department of \\ Histopathology, Bayero University \\ Kano, Aminu Kano Teaching hospital, \\ Kano, Nigeria
}

\begin{abstract}
Malignant histiocytosis is a rare condition with variable clinical presentation and prognosis. Its diagnosis requires high index of suspicion and further evaluation. Thus, it poses a diagnostic as well as management challenges especially in resource poor setting. The objective is to highlight the diagnosis and treatment challenges in the management of malignant histiocytosis so that physicians can diagnose it early and initiate appropriate treatment. Our patient is a 35year-old man who presented with chest wall mass, dyspnoea and right cervical lymphadenopathy He was initially being evaluated for lymphoma. Biopsy of the lymph nodes was done twice in different hospitals and that of the chest wall mass done once. Histological diagnosis was arrived at with the aid of immunohistochemistry three months after his first presentation to the hospital. The patient was then commenced on chemotherapy and showed remarkable improvement on the short-term basis. Malignant histiocytosis is a rare disease laden with management challenges. Despite that, its diagnosis and treatment can be achieved even in resource poor setting with high index of suspicion, team work and good laboratory support.
\end{abstract}

\section{Introduction}

Malignant histiocytosis of the lungs and chest wall is a rare condition with variable clinical presentation and prognosis. The diagnosis requires high index of suspicion and further evaluation and it poses a diagnostic as well as management challenges especially in resource poor setting. ${ }^{1}$

\section{Case Report}

We present a 35-year-old man referred to our centre from a tertiary hospital located about $160 \mathrm{~km}$ away with a 10 months history of slowly growing anterior chest wall mass followed 3 months later by dyspnoea which was initially on exertion and later at rest (dyspnoea score of 5). There was associated orthopnoea, dry cough, low-grade fever, weight loss and dysphagia. The chest wall mass later spontaneously ruptured and started discharging serous fluid. A right sided neck swelling then appeared about 2 months to presentation. Left tube thoracostomy was done for the patient 3 months earlier which drained serous fluid. He had significant history of second-hand smoking and a positive family history of breast cancer in his half-sister.

On examination he was chronically illlooking with a body mass index of 17.1 $\mathrm{kg} / \mathrm{m}^{2}$, Karnofsky performance status scale of $20 \%$ and in marked respiratory distress but was haemodynamically stable. He had multiple, matted, hard and non-tender right Supraclavicular lymphadenopathy and bilateral medial group of axillary lymph nodes. There was an anterior chest wall mass across the sternum about $30 \times 20 \mathrm{~cm}$ with soft and hard components and attached to the skin and the underlying bones. There were dull percussion notes and decreased air entry over the middle and lower chest zones which was more on the left. The liver and spleen were not enlarged. Lymphoma, chest wall tumour and tuberculosis were entertained as differential diagnoses.

Sputum Acid-Fast bacilli (AFB) and retroviral screening were both negative and full blood count, clotting profile, urea, electrolytes and creatinine and liver function were normal. Chest X-ray showed bilateral non-patchy opacities more on the left side (Figure 1) and a chest CT Scan showed isodensity occupying most of the left lung field and a chest wall mass involving the sternum and the anterior ribs with intra-thoracic extension (Figure 2). Test aspiration revealed free flowing serous pleural fluid bilaterally.

A biopsy of the right cervical lymph node at the referring hospital revealed adipose tissue, fibromuscular tissue and thickened vascular channels with areas showing lymphocytic infiltrates and histiocytes. A suspicion of malignancy was high and immunohistochemistry was advised. A repeat cervical lymph node biopsy at our centre was also not conclusive and a trucut biopsy of the chest wall mass was done to examine the two samples. The result showed infiltrating clusters and sheets of large ovoid cells with vesicular hyperchro-
Correspondence: Jameel Ismail Ahmad, Division of Cardiothoracic Surgery, Surgery Department, Bayero University Kano/Aminu Kano Teaching hospital, PMB 3452, Kano, Nigeria.

E-mail: iajameel@yahoo.com

Key words: Malignant histiocytosis, diagnosis, treatment, challenges, immunohistochemistry.

Contributions: the authors contributed equally.

Conflict of interest: the authors declare no potential conflict of interest.

Funding: none

Received for publication: 30 July 2018. Accepted for publication: 5 October 2018.

This work is licensed under a Creative Commons Attribution NonCommercial 4.0 License (CC BY-NC 4.0).

C) Copyright J.I. Ahmad et al., 2018

Licensee PAGEPress, Italy

Pyramid Journal of Medicine 2018; 1:9

doi:10.4081/pjm.2018.9

matic nuclei and abundant eosinophilic cytoplasm disposed within a fibrocollagenous tissue containing moderate infiltrates of neutrophils, eosinophils, lymphocytes and mast cells (Figure 3) and Immunohistochemistry showed $\mathrm{S}_{100}$ positivity (Figure 4). Thus, a histological diagnosis of malignant histiocytosis was then made.

Bone marrow, liver and Splenic involvements were excluded with bone marrow aspiration and abdominal ultrasound scan. We then made the diagnosis of multi-systemic Langerhan's cell malignant histiocytosis of the lung, chest wall and the lymphatic system.

Bilateral tube thoracostomy was done to drain the pleural effusions. The patient was prepared for chemotherapy which is the standard treatment and he was commenced on Vinblastine and prednisolone regimen as recommended by the Histiocyte Society evaluation and treatment guidelines 2009 (Vinblastine $6 \mathrm{mg} / \mathrm{m}^{2}=10 \mathrm{mg}$ weekly and Prednisolone $40 \mathrm{mg} / \mathrm{m}^{2}=60 \mathrm{mg}$ daily). Adjunctive treatments included antacids, Allupurinol, analgesics and antibiotics. He successfully completed the 7-weeks first course of the induction treatment and made remarkable improvement evidenced by regression of the chest wall mass and the right supraclavicular lymph nodes (Figure 5 ), improved dyspnoea score (from 5 to 1 ) and Karnofsky performance status scale (from $20 \%$ to $80 \%$ ) 
The 7-weeks second course of the induction treatment was commenced but the patient became less compliant as the support he receives from sponsoring relations dwindled and the course was also interrupted by episodes of anaemia, leucopenia and thrombocytopenia, generalized body rashes and ulceration of the chest wall mass. He then absconded but we traced him after 6 weeks and convinced him and the relations on the need to return for evaluation and commencement of maintenance treatment.

\section{Discussion}

Langerhan's cell histiocytosis is a rare condition especially in adults. The International Histiocyte registry reported 274 cases from 13 countries in 18 months. ${ }^{2}$ Pulmonary LCH is even rarer with a prevalence of 0.07-0.27/1,000,000 population. ${ }^{3}$ It is commoner among 20-40 years old.

The specific aetiology of pulmonary $\mathrm{LCH}$ is unknown but cigarette smoking is highly associated with it in about $50-90 \%$ of cases. ${ }^{2,4}$

The clinical presentation is variable from being asymptomatic to dyspnoea, cough, chest pain, soft tissue, bone swelling, weight loss, night sweats and fever. Spontaneous pneumothorax is reported in $10-20 \%$ of patients. ${ }^{5-8}$ Increased thirst and polyuria suggest hypothalamic and/or pituitary involvement.

Cytological and histological evaluation from Transthoracictrucut biopsy, bronchoalveolar lavageor Video assisted thoracic surgery biopsy is diagnostic. Presence of Birbick granules and immunohistochemical demonstration of Langerin (CD 207) and CD1a. $\mathrm{S}_{100}$ is an important marker although it can be found in macrophages and neuroendocrine cells. ${ }^{4,9}$

There are different classifications for LCH. However, the two commonly use are: World Health Organisation (WHO) classification and Histiocyte Society classification and Clinical classification which classified it into Single System LCH (SS-LCH) and Multi-System LCH (MS-LCH) as in Table
1. Systemic chemotherapy is the main stay of treatment of LCH but radiotherapy and hormonal therapy may also be used. Surgery has limited role. The indications for systemic therapy include SS-LCH with
"CNS-risk" lesions, SS-LCH with multifocal bone lesions (MFB), SS-LCH with "special site" lesions and MS-LCH with/without involvement of "risk organs". Treatment duration of 12 months is advocated over 6

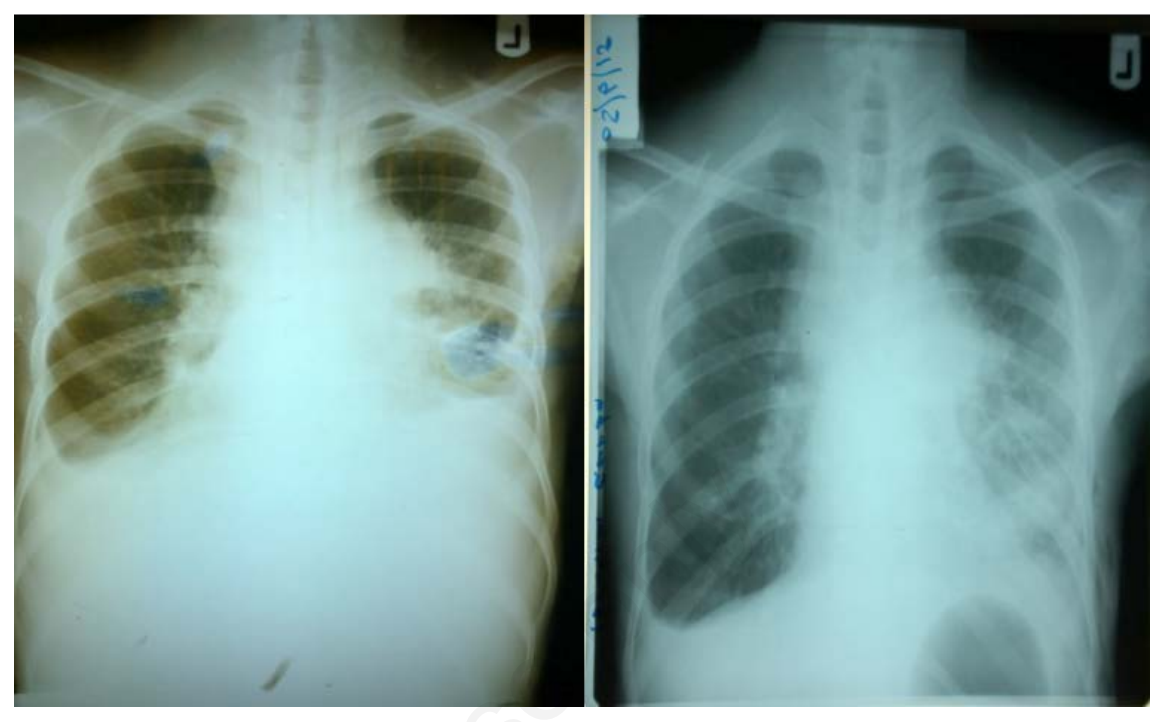

Figure 1. Chest X-ray for the patient with pulmonary malignant histiocytosis.

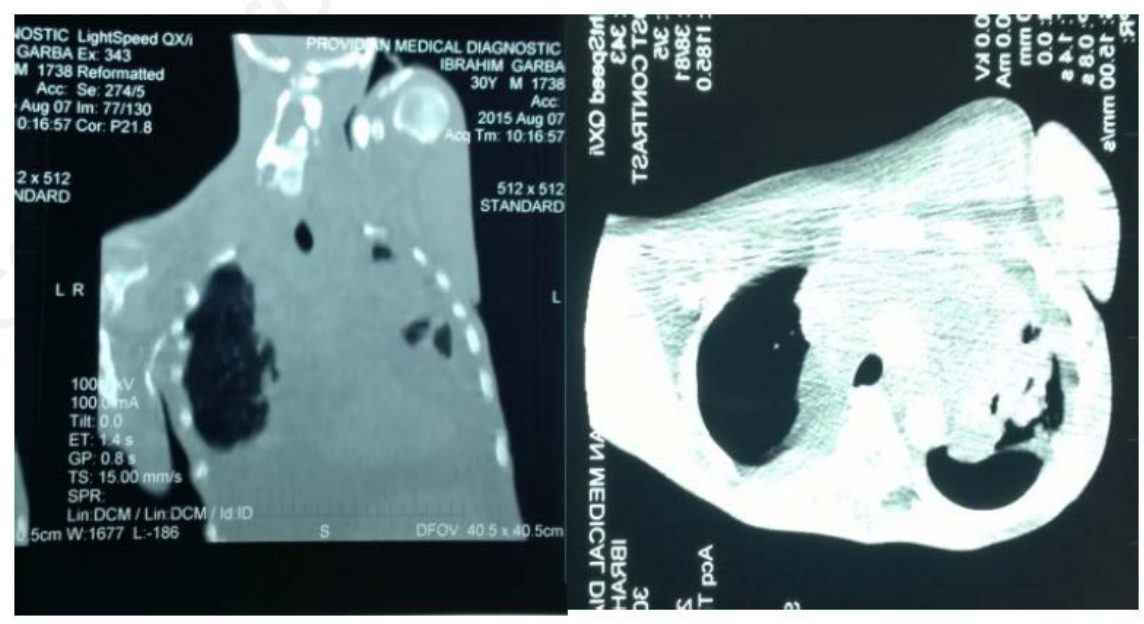

Figure 2. Chest computed tomography scan for the patient with pulmonary malignant histiocytosis.

Table 1. Two main classifications for Langerhans cell histiocytosis.

\section{Single System LCH (SS-LCH)}

One organ/system involved (uni- or multifocal).

Bone: unifocal (single bone) or multifocal (>1 bone).

Skin. Lymph node (not the draining lymph node of another LCH lesion).

Lungs.

Hypothalamic-pituitary/central nervous system.

Other (e.g. thyroid, thymus)

\section{Multisystem LCH (MS-LCH)}

Two or more organs/systems involved.

With or without involvement of "Risk Organs" 
months. A combination of prednisone (PRED) and vinblastine (VBL) has been proven to be effective treatment with minimal toxicity.

MS-LCH patients like ours can have a variable clinical course. Those with or without risk involvement who respond to standard initial therapy have an excellent chance of long-term survival while nonresponders have an unfavourable prognosis.

The treatment strategies for pulmonary LCH include cessation of smoking/exposure, systemic chemotherapy, systemic steroid therapy and Lung transplantation (in severe respiratory failure or major pul-
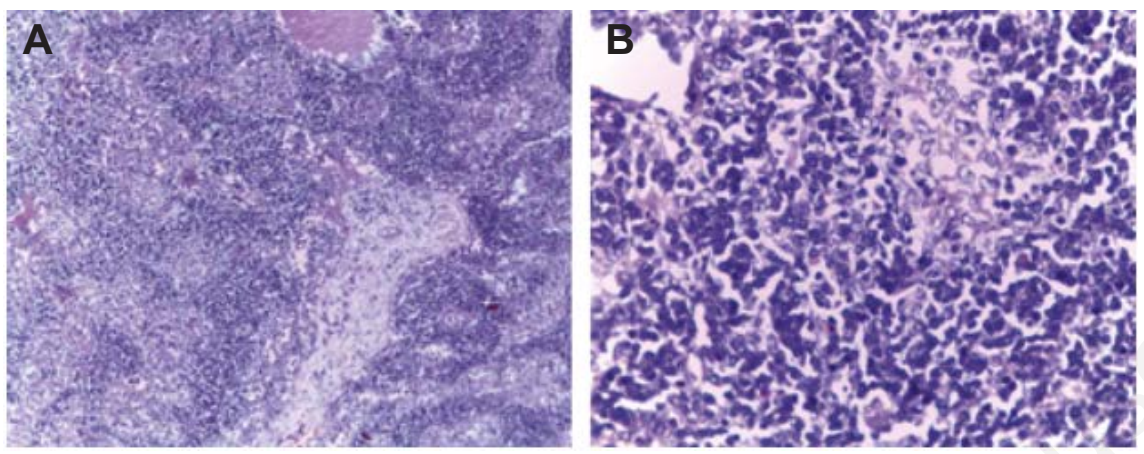

Figure 3. A) Section shows infiltrating nests of large ovoid-shaped Histiocytes disposed within a fibrocollageneous tissue containing moderate infiltrates of neutrophils, eosinophils, lymphocytes and mast cells. B) Section shows infiltrating nests and sheets of malignant Histiocytes with vesicular hyperchromatic nuclei, occasional prominent prominent nucleoli and abundant eosinophilic cytoplasm.
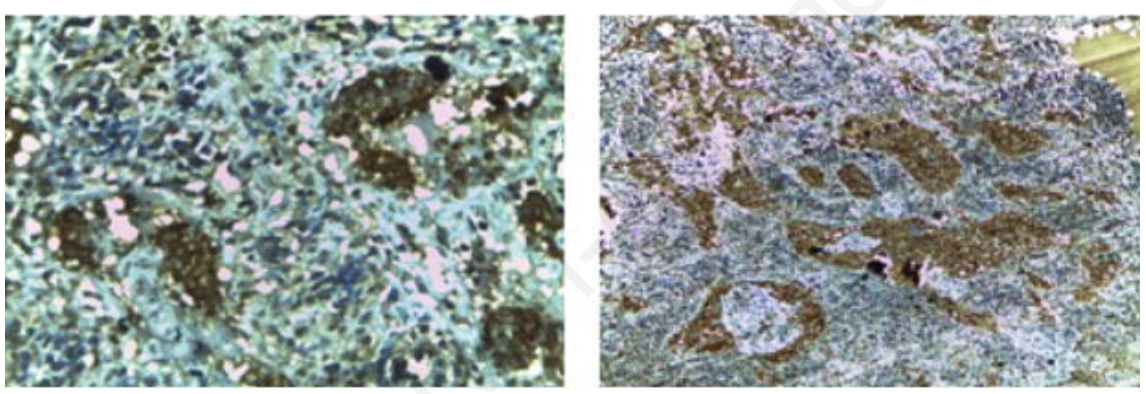

Figure 4. Immunohistochemistry section demonstrating S-100 positivity.

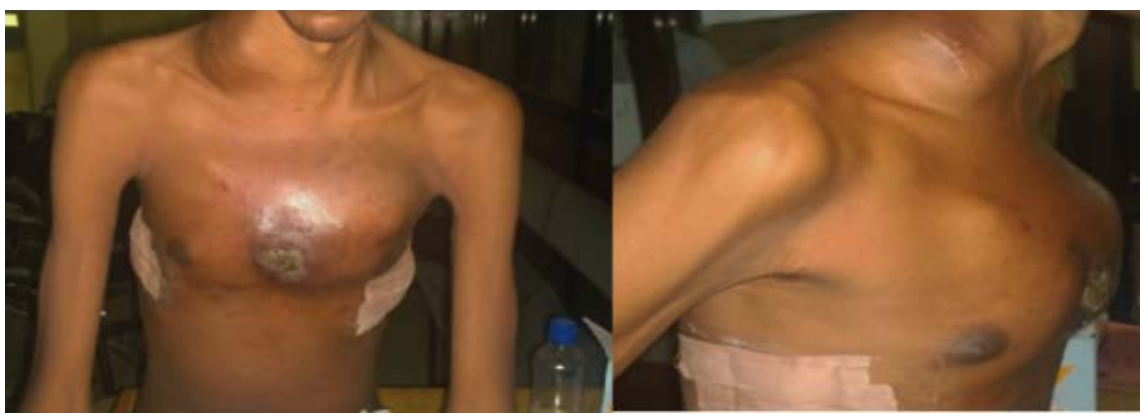

Figure 5. Decrease in size of chest wall mass following chemotherapy.

\section{References}

1. Histiocyte society. Langerhans cell histiocytosis: Histiocyte society evaluation and treatment guidelines. 2009. Available from: http//www.histiocytesociety.org.

2. Arico M, Girchikofsky M, Genereau T, et al. Langerhans cell histiocytosis in adults: report from the international registry of the histiocyte society. Eur J Cancer 2003;39:2341-8.

3. Watanabe R, Tatsumi K, Hashimoto S, et al. Clinico-pathological features of pulmonary Histiocytosis X. Intern Med 2001;40:998-1003.

4. Girchikofsky M, Arico M, Castillo D, et al. Management of adult patients with Langerhans cell histiocytosis: Recommendations from an expert panel on behalf of Euro-Histio-Net. Orphanate J Rare Dis 2013;8:72.

5. Tazi A. Adult pulmonary Langerhans cell histiocytosis. Eur Resp J 2006;27:1272-85.

6. Adam Z, Rehah Z, Koukalova R, et al. Pumonarylangerhans cell histiocytosisevaluation of the disease activity and treatment response using PET-CT (SUV max) Pulmo/SUV max Hepar index);Description of own experience and literature review. VnitrLek 2010;56:1228-5.

7. Bianchi M, Cataldi M. Pneumothorax secondary to pulmonary histiocytosis X. Minerva Chir 1999;54:531-6.

8. Kim CK, Park CB, Jun U, Seo EJ. Pulmonary langerhans cell histiocytosis presented with recurrent pneumothorax. Interac Cardiovasc Thorac Surg 2006;5:512-3.

9. Adam Z, Pour L, Krejci M, et al. Langerhans cell histiocytosis in adult patients: A disease with many faces: experience of a centre and an overview of the disease symptoms. Vnitr Lek 2008;54:1063-80. 